Supporting Information

\title{
Elucidating the Nature of the $\mathrm{Cu}(\mathrm{I})$ Active Site in $\mathrm{CuO} / \mathrm{TiO}_{2}$ for Excellent Low-Temperature $\mathrm{CO}$ Oxidation
}

Yarong Fang ${ }^{\dagger}$, Xiao Chi ${ }^{\sharp \ddagger}$, Li Li $i^{\dagger}$, Ji Yang ${ }^{\dagger}$, Shoujie Liu ${ }^{\dagger}$, Xingxu Lu ${ }^{\S}$, Wen Xiao ${ }^{\|}$, Liming Wang ${ }^{\perp}$, Zhu Luo $^{* \dagger}$, Weiwei Yang ${ }^{\dagger}$, Siyu Hu ${ }^{\dagger}$, Juxia Xiong ${ }^{\dagger}$, Son Hoang ${ }^{\dagger}$, Hongtao Deng ${ }^{\dagger}$, Fudong Liu ${ }^{\#}$, Lizhi Zhang ${ }^{\dagger}$, Puxian Gao *\$, Jun Ding", Yanbing Guo *广

'Key Laboratory of Pesticide \& Chemical Biology of Ministry of Education, Institute of Environmental and Applied Chemistry, College of Chemistry, Central China Normal University, Wuhan 430079, P. R. China

"Singapore Synchrotron Light Source National University of Singapore 5 Research Link, 117603, Singapore

${ }^{\S}$ Department of Chemical, Materials and Biomolecular Engineering, Institute of Materials Science, University of Connecticut, Storrs, CT 06269-3136, USA

"Department of Materials Science and Engineering, National University of Singapore, 117575, Singapore

${ }^{\perp}$ CAS Key Laboratory for Biomedical Effects of Nanomaterials \& Nanosafety, Institute of High Energy Physics Department of Materials Science and Engineering Chinese Academy of Sciences, Beijing 100049, China

\#Department of Civil, Environmental, and Construction Engineering, Catalysis Cluster for Renewable Energy and Chemical Transformations (REACT), NanoScience Technology Center, University of Central Florida, Orlando, FL 32816, USA

* Correspondence to Prof. Luo (Email: luo.z@mail.ccnu.edu.cn); Prof. Gao (Email: puxian.gao@ucconn.edu); Prof. Guo (Email: guoyanbing@mail.ccnu.edu.cn)

11 pages, 17 figures, 4 tables 


\section{CONTENTS}

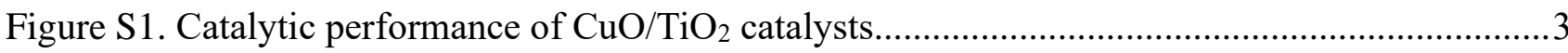

Figure $\mathrm{S} 2$. The $\mathrm{BET}$ characterization of $\mathrm{CuO} / \mathrm{TiO}_{2}$ catalysts ...................................................... 3

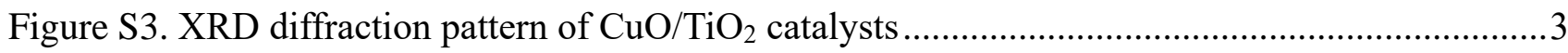

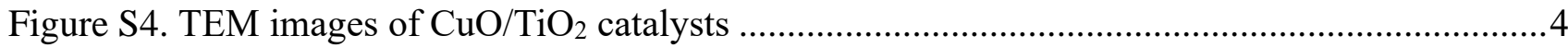

Figure S5. EDX elemental mapping of $\mathrm{Cu}$, Ti on a selected region of TEM image of $\mathrm{CuO} / \mathrm{TiO}_{2}$ catalysts

4

Figure S6. EDX elemental mapping of $\mathrm{Cu}$, $\mathrm{Ti}$ on $\mathrm{CuO}-\mathrm{TiO}_{2}$ interface and $\mathrm{HRTEM}$ image of the interfacial structure

Figure S7. The XPS and $\mathrm{Cu} \mathrm{LMM}$ survey of $\mathrm{CuO} / \mathrm{TiO}_{2}$ catalysts .................................................5

Figure S8. Ti L-edge XANES spectra of $\mathrm{CuO} / \mathrm{TiO}_{2}$ catalysts $\quad$................................................. 6

Figure $\mathrm{S} 9$. Oxygen based temperature programmed desorption $\left(\mathrm{O}_{2}-\mathrm{TPD}\right)$ of $\mathrm{CuO} / \mathrm{TiO}_{2}$ catalysts $\quad \ldots .6$

Figure $\mathrm{S} 10$. DRIFT spectra of $\mathrm{Cu}_{2} \mathrm{O}, \mathrm{CuO}$ and $\mathrm{Cu}$ as references

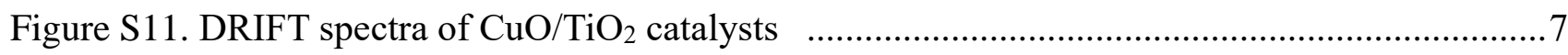

Figure S12. The time-on-stream DRIFT spectra under pure CO condition ..................................

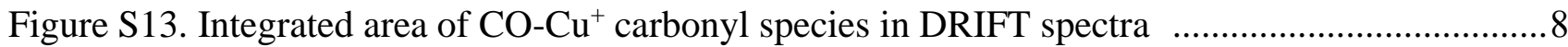

Figure S14. The time-on-stream DRIFT spectra under ultra-low CO concentration ........................8

Figure $\mathrm{S} 15 . \mathrm{CO}$ signal of $\mathrm{CuO} / \mathrm{TiO}_{2}$ catalysts in $\mathrm{CO}_{\mathrm{x}}-\mathrm{TPD}$ process .......................................

Figure S16. The primitive cell of the model of interfacial structure

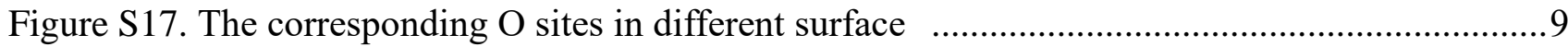

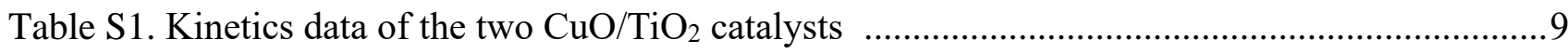

Table S2. Multiple linear regression results of the measured kinetic data ...................................10

Table S3. Catalytic activity comparison of the recently reported CO oxidation catalysts ...............10

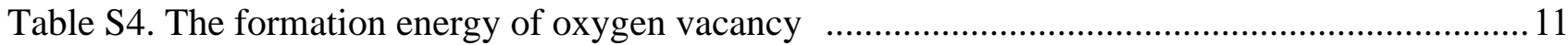




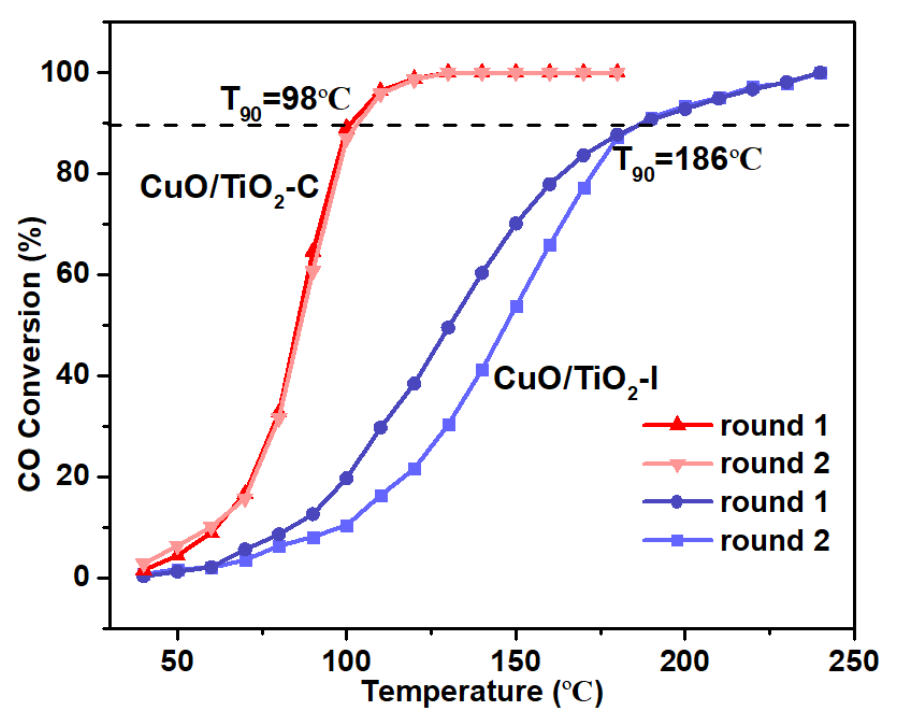

Figure S1. Light-off curves of $\mathrm{CuO} / \mathrm{TiO}_{2}-\mathrm{C}$ and $\mathrm{CuO} / \mathrm{TiO}_{2}-\mathrm{I}$ samples. Reaction gas consist of $1 \% \mathrm{CO}, 20 \% \mathrm{O}_{2}$ and balanced with $\mathrm{N}_{2}$.

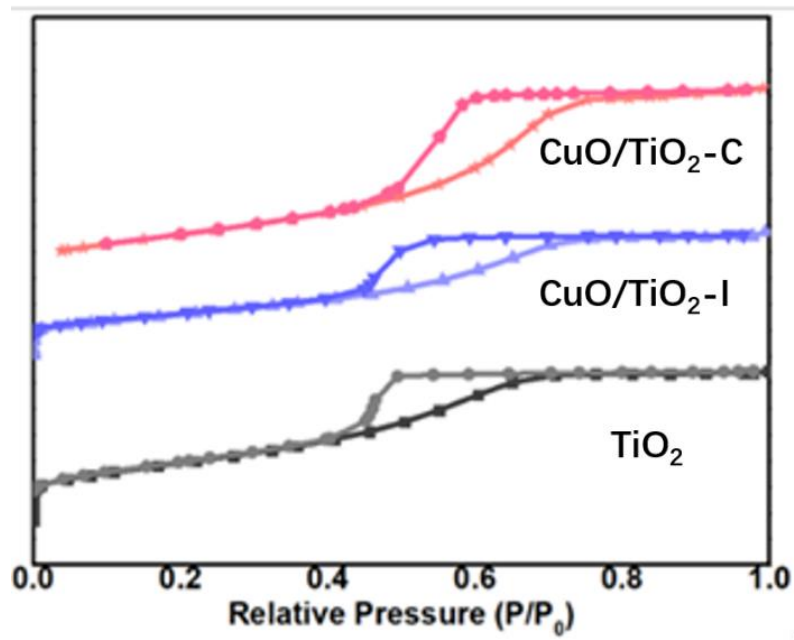

Figure S2. $\mathrm{N}_{2}$ adsorption-desorption isotherms of $\mathrm{CuO} / \mathrm{TiO}_{2}-\mathrm{C}, \mathrm{CuO} / \mathrm{TiO}_{2}-\mathrm{I}$ and $\mathrm{TiO}_{2}$ samples.

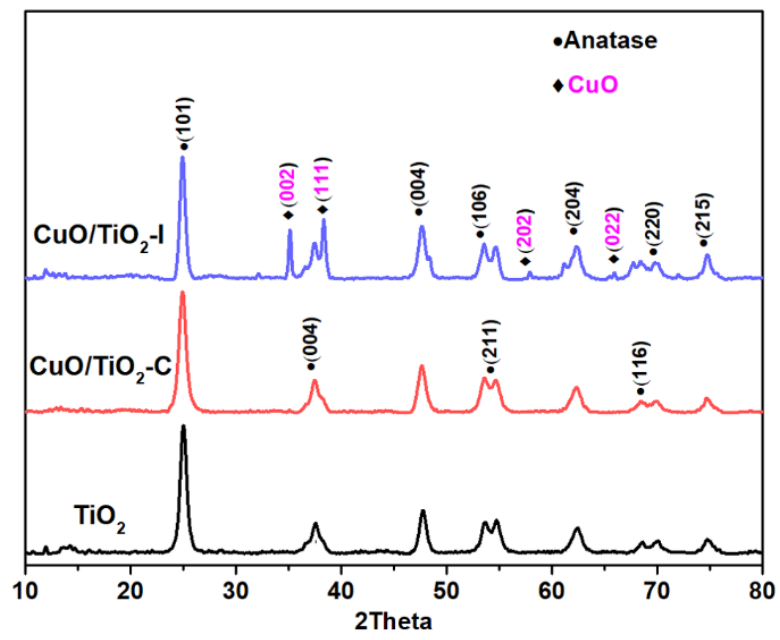

Figure S3. XRD patterns of $\mathrm{CuO} / \mathrm{TiO}_{2}-\mathrm{C}, \mathrm{CuO} / \mathrm{TiO}_{2}-\mathrm{I}$ and $\mathrm{TiO}_{2}$ samples. 

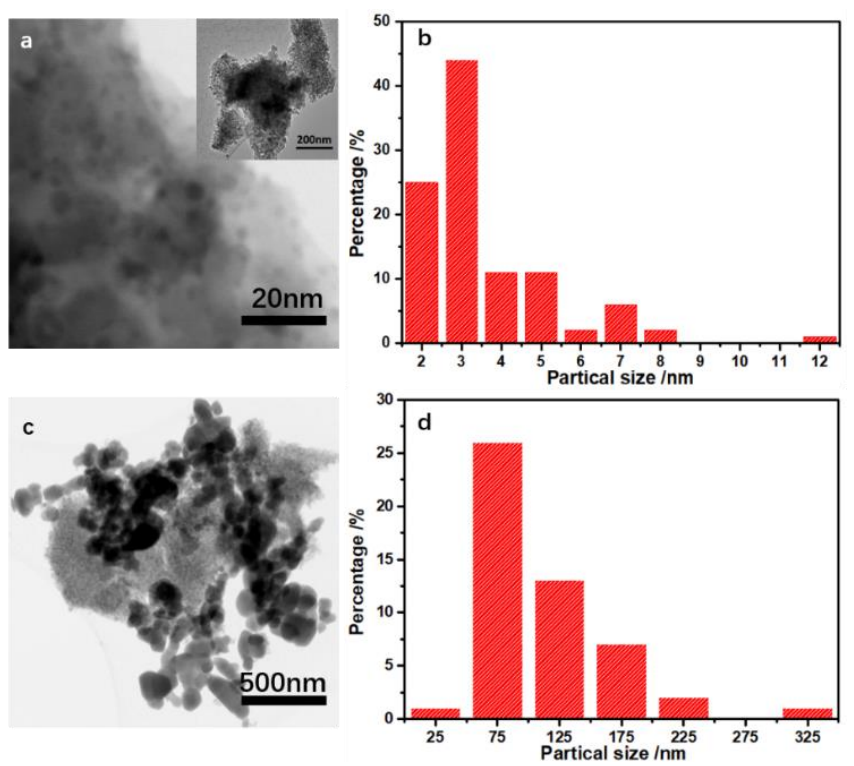

Figure S4. TEM bright field micrographs of a: $\mathrm{CuO} / \mathrm{TiO}_{2}-\mathrm{C}$ and $\mathbf{c}$ : $\mathrm{CuO} / \mathrm{TiO}_{2}-\mathrm{I}$; corresponding particle size distribution for b: $\mathrm{CuO} / \mathrm{TiO}_{2}-\mathrm{C}$ and $\mathbf{d}: \mathrm{CuO} / \mathrm{TiO}_{2}-\mathrm{I}$.
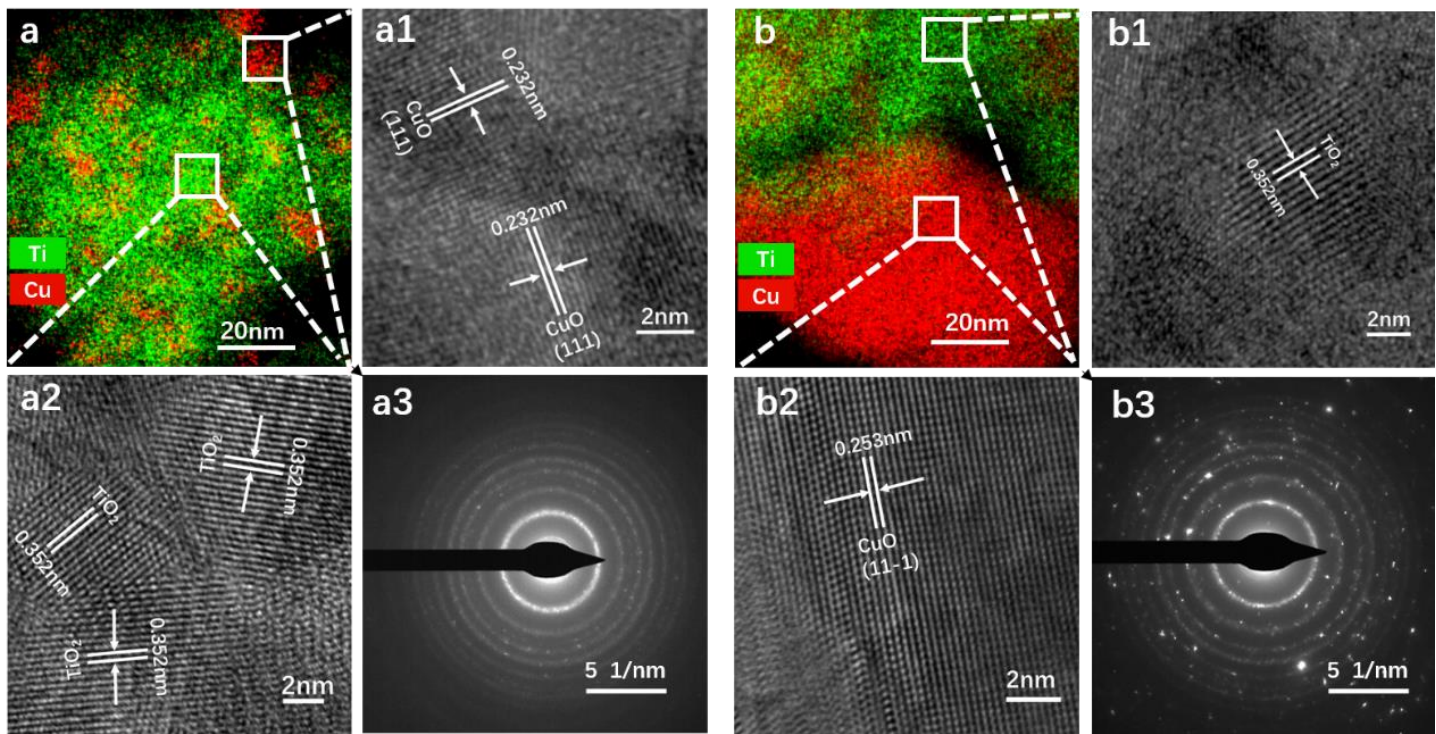

Figure S5. a: TEM-EDS mapping of $\mathrm{CuO} / \mathrm{TiO}_{2}-\mathrm{C}$ sample and a1, a2: the corresponding HRTEM images; a3: SAED pattern taken from the square region; b: TEM-EDS mapping of $\mathrm{CuO} / \mathrm{TiO}_{2}-\mathrm{I}_{\text {sample }}$ and b1, b2: the corresponding HRTEM image; b3: SAED pattern taken from the square region. 


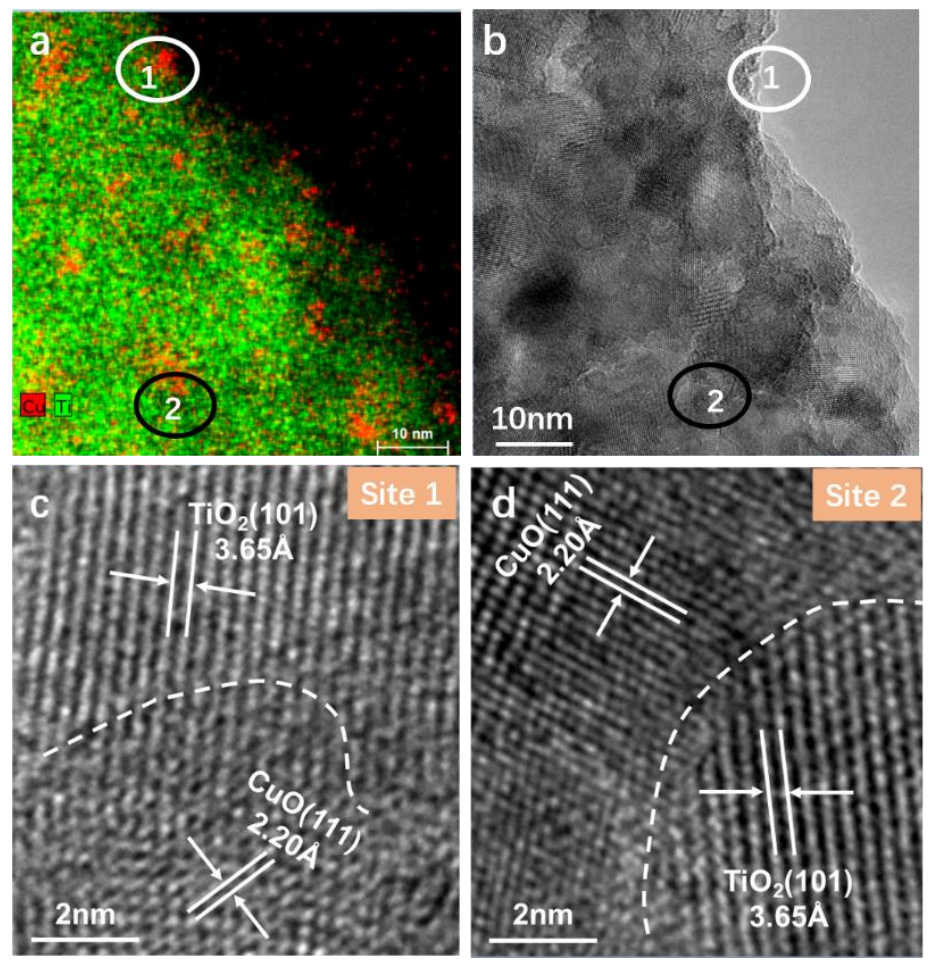

Figure S6. a: TEM-EDS mapping of $\mathrm{CuO} / \mathrm{TiO}_{2}-\mathrm{C}$ sample; b: the corresponding HRTEM images; enlarged HRTEM image taken from the c: circled region 1 and $\mathbf{d}$ : circled region 2.

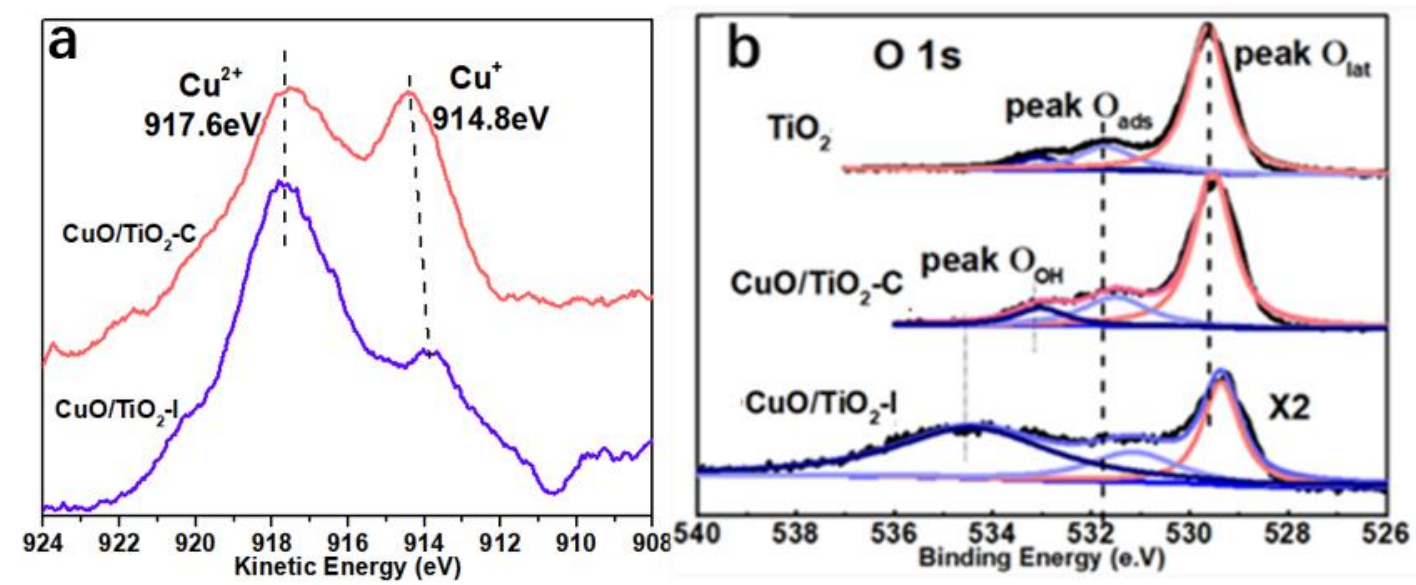

Figure S7. XPS spectra for $\mathrm{CuO} / \mathrm{TiO}_{2}-\mathrm{C}, \mathrm{CuO} / \mathrm{TiO}_{2}-\mathrm{I}$ and $\mathrm{TiO}_{2}$ samples for a: Auger $\mathrm{Cu} \mathrm{LMM}$ spectra for $\mathrm{CuO} / \mathrm{TiO}_{2}-\mathrm{C}, \mathrm{CuO} / \mathrm{TiO}_{2}-\mathrm{I}$ and $\mathrm{TiO}_{2}$ samples; b: $\mathrm{O} 1$ s. 


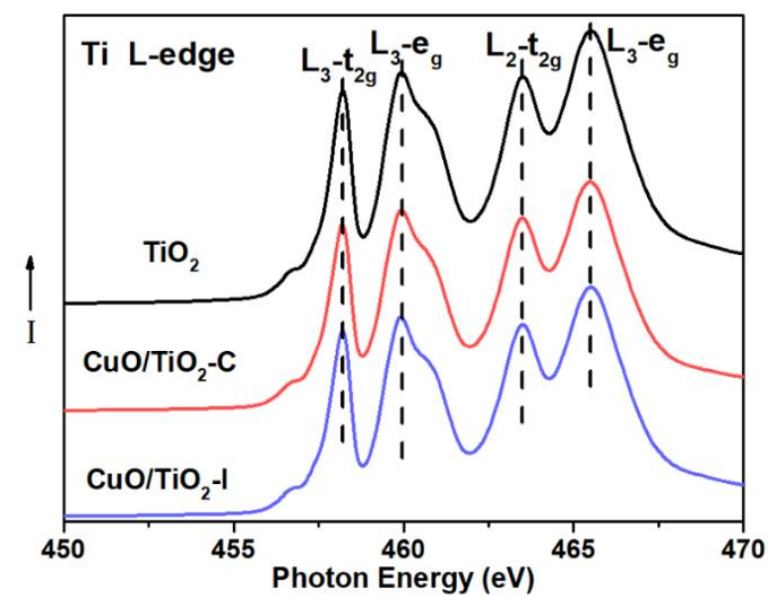

Figure S8. Ti L-edge of XANES spectra of $\mathrm{CuO} / \mathrm{TiO}_{2}-\mathrm{C}, \mathrm{CuO} / \mathrm{TiO}_{2}-\mathrm{I}$ and $\mathrm{TiO}_{2}$ samples.

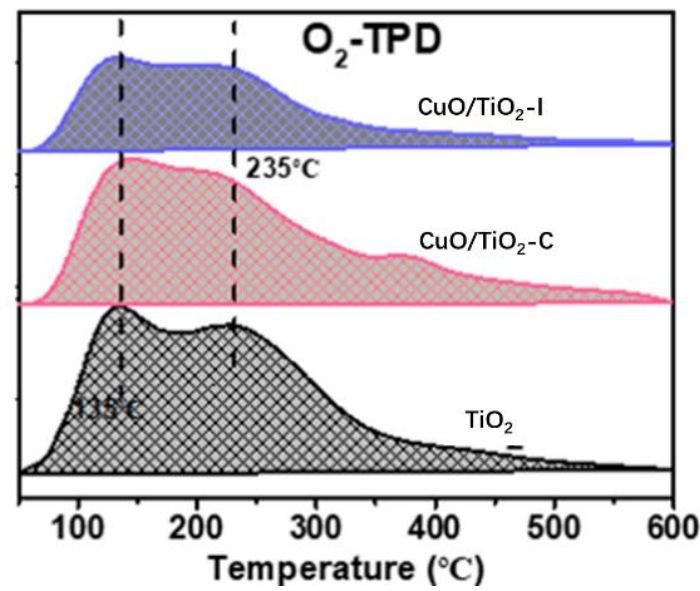

Figure S9. Oxygen based temperature programmed desorption $\left(\mathrm{O}_{2}-\mathrm{TPD}\right)$ of $\mathrm{CuO} / \mathrm{TiO}_{2}$ catalysts.

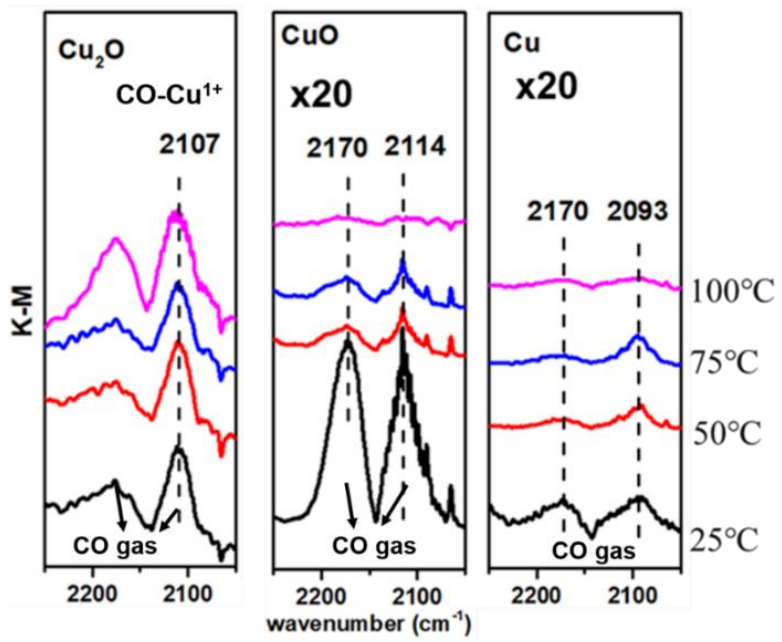

Figure S10. DRIFT spectra as a function of temperature under simple CO atmosphere: total flow: 50 sccm; $\mathrm{CO}: 0.5 \mathrm{sccm}$; balance: $\mathrm{N}_{2}$ for $\mathrm{Cu}_{2} \mathrm{O}, \mathrm{CuO}$ and $\mathrm{Cu}$ powder, respectively. 

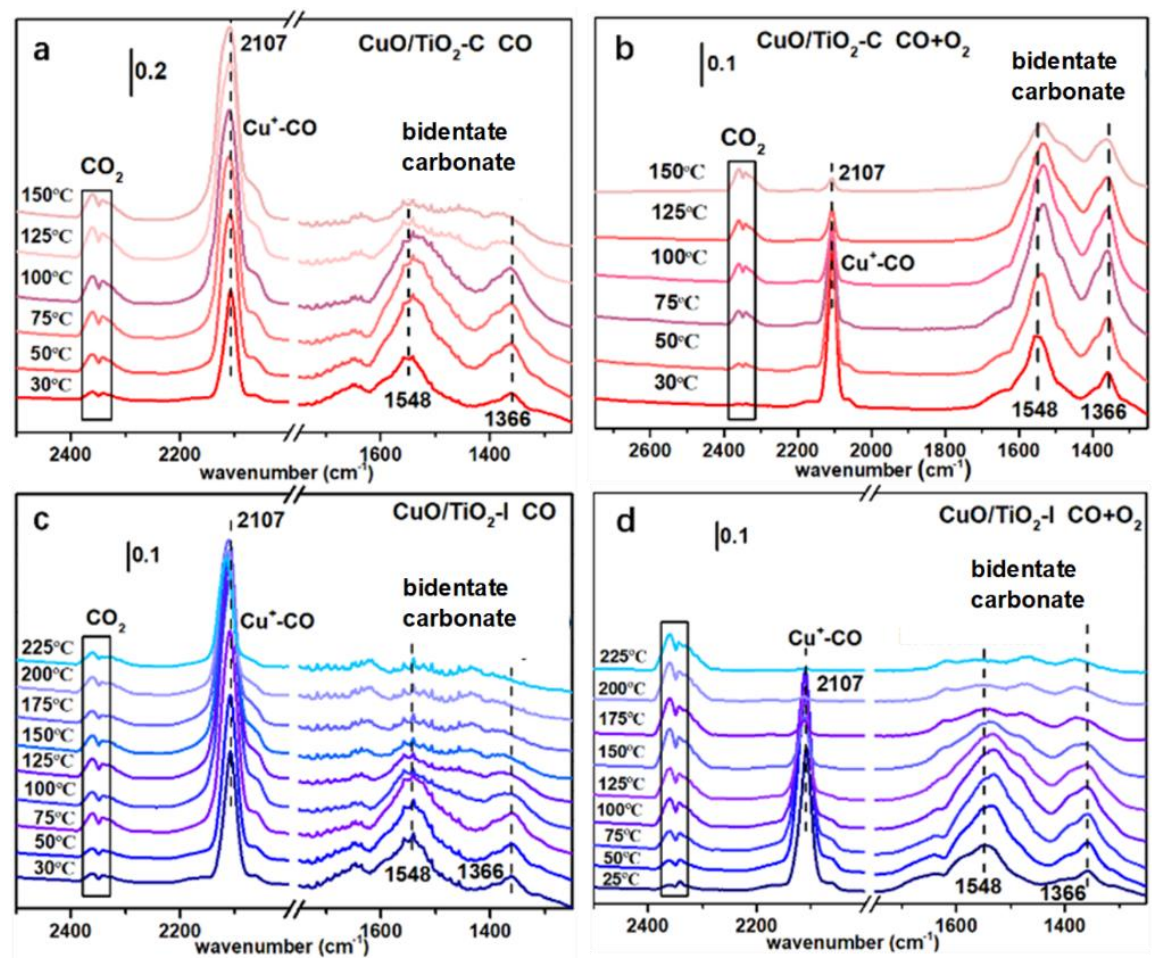

Figure S11. DRIFT spectra as a function of temperature under simple CO atmosphere: total flow: 50 sccm; $\mathrm{CO}: 0.5 \mathrm{sccm}$; balance: $\mathrm{N}_{2}$ for a: $\mathrm{CuO} / \mathrm{TiO}_{2}-\mathrm{C}$ and c: $\mathrm{CuO} / \mathrm{TiO}_{2}-\mathrm{I}$ catalysts, respectively; and under $\mathrm{CO}$ oxidation conditions total flow: $50 \mathrm{sccm}$; $\mathrm{CO}: 0.5 \mathrm{sccm} ; \mathrm{O}_{2}: 2.5 \mathrm{sccm}$; balance: $\mathrm{N}_{2}$ for b: $\mathrm{CuO} / \mathrm{TiO}_{2}-\mathrm{C}$ and $\mathbf{d}: \mathrm{CuO} / \mathrm{TiO}_{2}-\mathrm{I}$ catalysts, respectively.
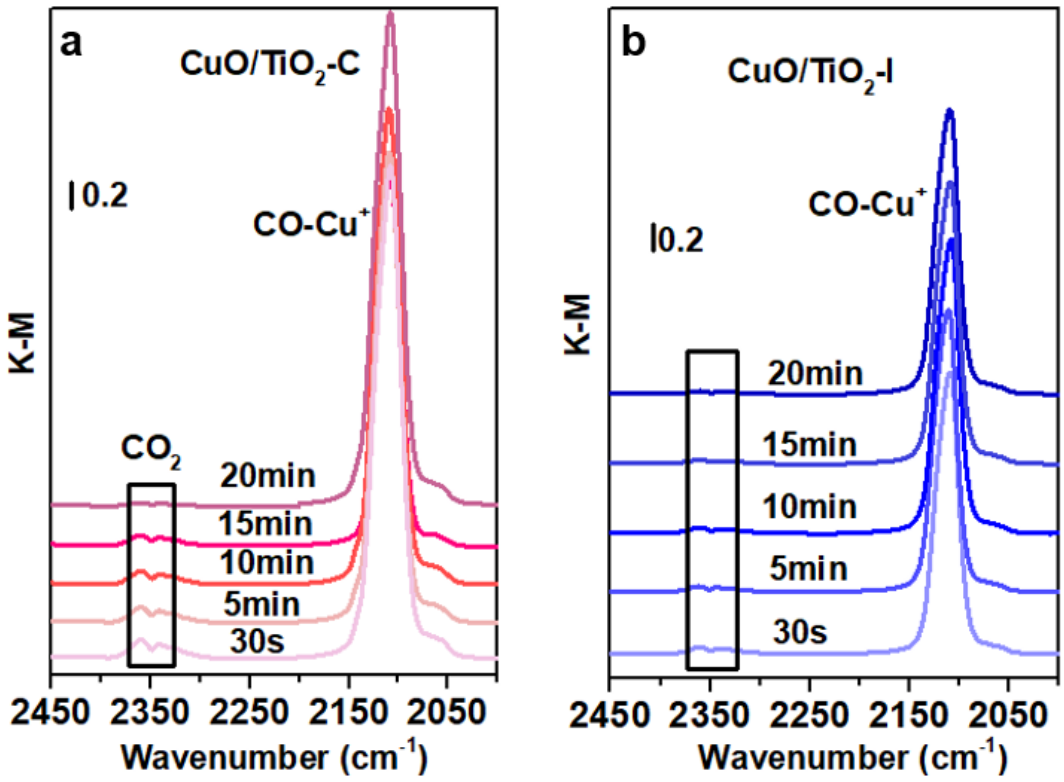

Figure S12. DRIFT spectra as a function of time under simple $\mathrm{CO}$ atmosphere at $100^{\circ} \mathrm{C}$ for $\mathbf{a}$ : $\mathrm{CuO} / \mathrm{TiO}_{2}-\mathrm{C}$ and b: $\mathrm{CuO} / \mathrm{TiO}_{2}-\mathrm{I}$ catalysts. Reaction condition: total flow: $50 \mathrm{sccm} ; \mathrm{CO}: 0.5 \mathrm{sccm}$; balance: $\mathrm{N}_{2}$. 


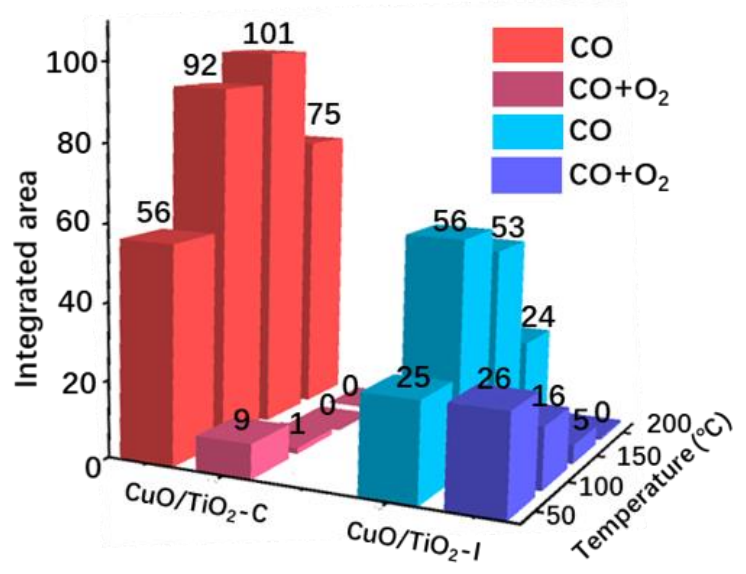

Figure S13. Integrated area of the $\mathrm{Cu}^{+}-\mathrm{CO}$ carbonyl at $2107 \mathrm{~cm}^{-1}$.
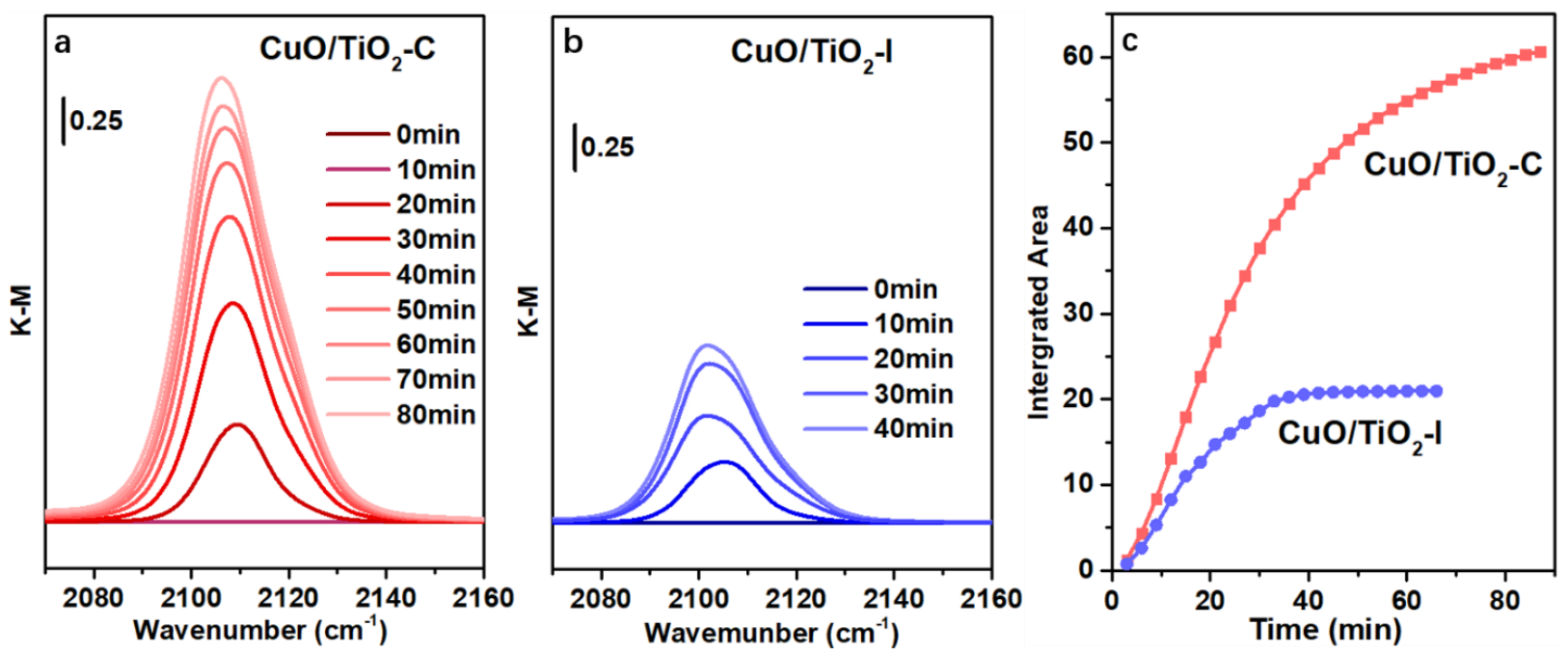

Figure S14. DRIFT spectra as a function of time under simple $\mathrm{CO}$ atmosphere at $50^{\circ} \mathrm{C}$ for $\mathbf{a}$ :

$\mathrm{CuO} / \mathrm{TiO}_{2}-\mathrm{C}$ and b: $\mathrm{CuO} / \mathrm{TiO}_{2}-\mathrm{I}$ catalysts; $\mathbf{c}$ : Integrated area of the $\mathrm{Cu}^{+}-\mathrm{CO}$ carbonyl band at 2107 $\mathrm{cm}^{-1}$ as a function of the adsorption time. Reaction condition: total flow: 50sccm; CO: 200ppm; balance: $\mathrm{N}_{2}$.

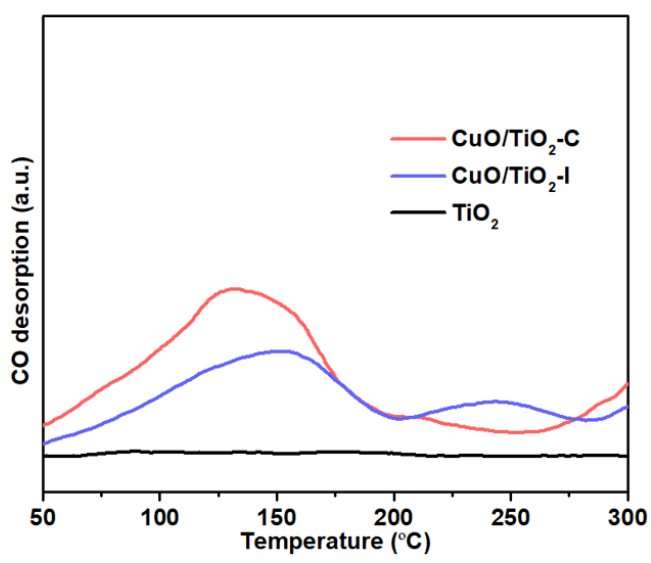

Figure S15. Traced $\mathrm{CO}$ signal of the two $\mathrm{CuO} / \mathrm{TiO}_{2}$ catalysts during the $\left(\mathrm{CO}_{\mathrm{x}}-\mathrm{TPD}\right)$ process. 

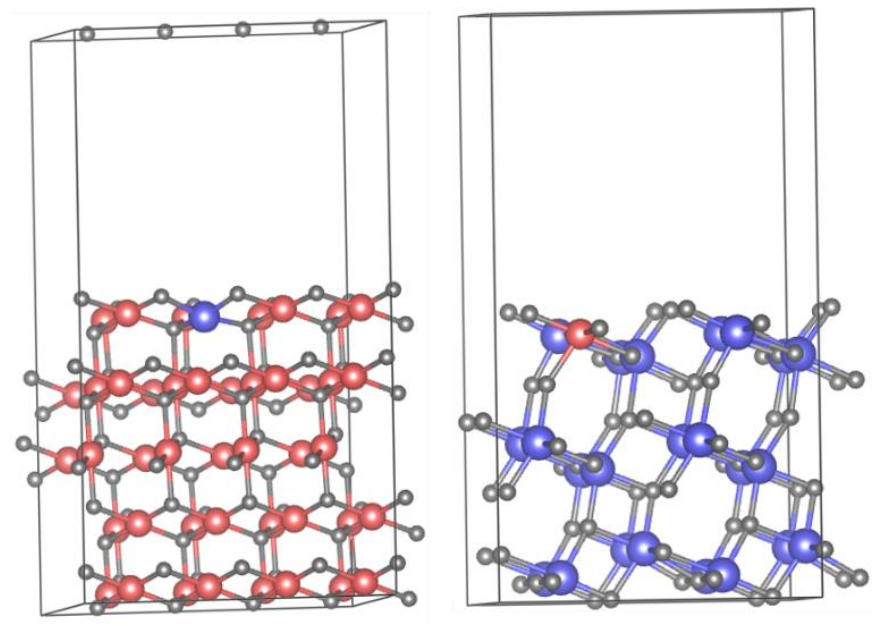

Figure S16. The primitive cell of left:Ti doped $\mathrm{CuO}(111)$ and right: $\mathrm{Cu}$ doped $\mathrm{TiO}_{2}(101)$.

a

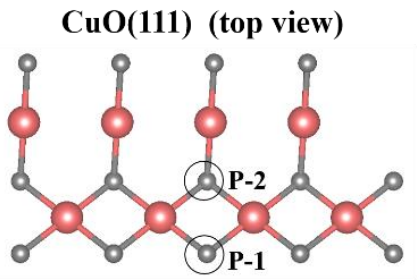

b Ti doped $\mathrm{CuO}(111)$ (top view)

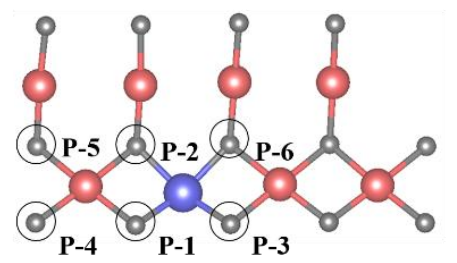

$\mathrm{c}$

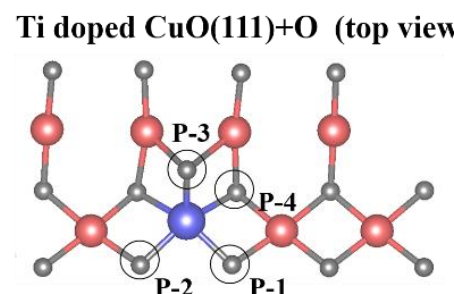

Figure S17. The corresponding O sites in different surface.

Table S1. Measured $\mathrm{CO}$ reaction rate $\left(\mathrm{r}_{\mathrm{CO}}\right)$ of the two $\mathrm{CuO} / \mathrm{TiO}_{2}$ catalysts at different gas conditions.

\begin{tabular}{|c|c|c|c|c|c|c|}
\hline \multirow[b]{2}{*}{ No } & \multicolumn{3}{|c|}{$\mathrm{CuO} / \mathrm{TiO}_{2}-\mathrm{C}$} & \multicolumn{3}{|c|}{$\mathrm{CuO} / \mathrm{TiO}_{2}-\mathrm{I}$} \\
\hline & $\begin{array}{c}P_{\mathrm{CO}} \\
(\mathrm{kPa})\end{array}$ & $\begin{array}{c}P_{\mathrm{O} 2} \\
(\mathrm{kPa})\end{array}$ & $\begin{array}{c}r_{\mathrm{CO}} \times 10^{7} \\
\left(\mathrm{~mol} \mathrm{~m}_{\mathrm{cat}}^{-2} \mathrm{~s}^{-1}\right)\end{array}$ & $\begin{array}{c}P_{\mathrm{CO}} \\
(\mathrm{kPa})\end{array}$ & $\begin{array}{c}P_{\mathrm{O} 2} \\
(\mathrm{kPa})\end{array}$ & $\begin{array}{c}r_{\mathrm{CO}} \times 10^{7} \\
\left(\mathrm{~mol} \mathrm{~m}_{c a t}^{-2} \mathrm{~s}^{-1}\right)\end{array}$ \\
\hline 1 & 1 & 0.25 & 2.83 & 1 & 0.25 & 1.73 \\
\hline 2 & 0.75 & 0.5 & 2.15 & 0.75 & 0.5 & 1.42 \\
\hline 3 & 0.5 & 0.75 & 1.74 & 0.5 & 0.75 & 1.08 \\
\hline 4 & 0.25 & 1 & 1.13 & 0.25 & 1 & 0.61 \\
\hline
\end{tabular}


Table S2. Fitting results of the measured CO reaction rate $\left(\mathrm{r}_{\mathrm{CO}}\right)$.

\begin{tabular}{cccc}
\hline Catalysts & $a$ & $b$ & $A$ \\
\hline $\mathrm{CuO} / \mathrm{TiO}_{2}-\mathrm{C}$ & 0.65 & 0.005 & $5.39 \times 10^{-5}$ \\
$\mathrm{CuO} / \mathrm{TiO}_{2}-\mathrm{I}$ & 0.77 & 0.008 & $6.17 \times 10^{-5}$ \\
\hline
\end{tabular}

The reaction order with respect to $\mathrm{CO}(\mathrm{a})$ and $\mathrm{O}_{2}$ (b) were obtained by fitting the four measured plots of rates $\left(\mathrm{r}_{\mathrm{CO}}\right)$ in Table $\mathbf{S 1}$ according to the following equation: $\ln \mathrm{r}=-\frac{\mathrm{Ea}}{\mathrm{RT}}+\ln \mathrm{A}+\mathrm{a} \ln \left[C_{\mathrm{CO}}\right]+\mathrm{b} \ln \left[C_{\mathrm{O} 2}\right]$

Table S3. Comparison of the recently studied CO oxidation catalysts containing copper species as active site or titanium dioxide as support.

\begin{tabular}{|c|c|c|c|c|c|c|}
\hline Catalysts & $\begin{array}{c}\text { BET } \\
\text { surface } \\
\text { area } \\
\left(\mathrm{m}^{2} / \mathrm{g}\right)\end{array}$ & $\begin{array}{c}\text { Space } \\
\text { velocity } \\
(\mathbf{m L} /(g \cdot h))\end{array}$ & $\begin{array}{r}\mathbf{T}_{\mathbf{5 0}} \\
\left({ }^{\circ} \mathbf{C}\right)\end{array}$ & $\begin{array}{l}\mathbf{T}_{100} \\
\left({ }^{\circ} \mathrm{C}\right)\end{array}$ & $\begin{array}{c}\mathbf{E}_{\text {vac }} \\
(\mathrm{kJ} / \mathrm{mol})\end{array}$ & Reference \\
\hline $\mathrm{CuO} / \mathrm{TiO}_{2}-\mathrm{C}$ & 42 & 60,000 & 76 & 120 & 27.6 & This work \\
\hline $\mathrm{CuO} / \mathrm{TiO}_{2}$ & -- & 30,000 & -- & -- & 26.3 & $\begin{array}{c}\text { Langmuir, 2012, 28, } \\
\text { 9996-10002 }\end{array}$ \\
\hline $\mathrm{CuO}_{\mathrm{x}}-\mathrm{CeO}_{2}$ & 12.6 & 16,000 & 77 & 120 & -- & $\begin{array}{c}\text { ACS Catal. 2019, 9, } \\
\text { 2177-2195 }\end{array}$ \\
\hline $\begin{array}{c}\mathrm{Cu} \text { doped } \\
\mathrm{CeO}_{2}\end{array}$ & 66.4 & 8,000 & -- & 100 & 55.4 & $\begin{array}{c}\text { Nanoscale, } 2017,9, \\
8007-8014\end{array}$ \\
\hline $10 \mathrm{CuCe}-\mathrm{L}$ & 48 & 60,000 & 103 & 140 & -- & $\begin{array}{c}\text { Nanoscale, } 2018,10 \\
22775-22786\end{array}$ \\
\hline $\mathrm{Cu} / \mathrm{CeO}_{2}$ & -- & 60,000 & 83 & 142 & 75.1 & $\begin{array}{c}\text { ACS Catal. 2018, 8, } \\
5261-5275\end{array}$ \\
\hline $\mathrm{CuO}$ NPs & -- & 72,000 & -- & 180 & -- & $\begin{array}{l}\text { ACS Appl. Mater. } \\
\text { Interfaces, 2017, 9, } \\
\text { 2495-2499 }\end{array}$ \\
\hline $\mathrm{Cu}_{2} \mathrm{O} \mathrm{NPs}$ & -- & 15,000 & -- & 170 & -- & $\begin{array}{c}\text { Appl. Catal. B: } \\
\text { Environmental 2019, } \\
253,160-169\end{array}$ \\
\hline $\begin{array}{c}\mathrm{CCC}(\mathrm{CuO}- \\
\mathrm{Co}_{2} \mathrm{O}_{3-} \\
\left.\mathrm{CeO}_{2}\right)\end{array}$ & 41.7 & 150,000 & -- & 200 & -- & $\begin{array}{l}\text { Angew. Chem. Int. Ed. } \\
2015,54,13263-13267\end{array}$ \\
\hline $\mathrm{Pt} / \mathrm{TiO}_{2}(101)$ & 91 & 360,000 & 92 & 110 & -- & $\begin{array}{c}\text { ACS Catal. 2016, 6, } \\
7799-7809 .\end{array}$ \\
\hline $\mathrm{Pt} / \mathrm{TiO}_{2}$ & -- & 1333,000 & -- & 120 & 49 & $\begin{array}{c}\text { J. Am. Chem. Soc. } \\
\text { 2015, 137, } \\
10156-10159 \\
\end{array}$ \\
\hline $\mathrm{Au} / \mathrm{TiO}_{2}$ & -- & -- & -- & 125 & -- & $\begin{array}{c}\text { Angew. Chem. Int. Ed. } \\
2017,56,9597-9602\end{array}$ \\
\hline
\end{tabular}


Table S4. Formation energy of oxygen vacancy in different sites.

\begin{tabular}{|c|c|c|c|}
\hline \multirow{2}{*}{ Surface model } & \multirow{2}{*}{ O positions } & \multicolumn{2}{|c|}{$\mathrm{E}_{\mathrm{f}}\left(\mathrm{O}_{\mathrm{vac}}\right) / \mathrm{eV}$} \\
\hline & & $\mathrm{O}_{\text {rich }}$ & $\mathrm{O}_{\text {poor }}$ \\
\hline \multirow{2}{*}{$\mathrm{CuO}(111)$} & P-1 & 2.750 & 1.151 \\
\hline & P-2 & 2.880 & 1.281 \\
\hline \multirow{6}{*}{$\begin{array}{l}\text { Ti doped } \\
\mathrm{CuO}(111)\end{array}$} & P-1 & 2.671 & 1.072 \\
\hline & $\mathrm{P}-2$ & 3.370 & 1.771 \\
\hline & P-3 & 2.830 & 1.231 \\
\hline & $\mathrm{P}-4$ & 2.701 & 1.102 \\
\hline & P-5 & 2.861 & 1.262 \\
\hline & P-6 & 2.832 & 1.233 \\
\hline \multirow{4}{*}{$\begin{array}{c}\text { Ti doped } \\
\mathrm{CuO}(111)+\mathrm{O}\end{array}$} & P-1 & 2.682 & 1.083 \\
\hline & P-2 & 2.842 & 1.243 \\
\hline & P-3 & 2.664 & 1.065 \\
\hline & P-4 & 2.728 & 1.129 \\
\hline
\end{tabular}

In $\mathrm{O}_{\text {rich }}$ condition, the $\mu \mathrm{o}$ is given by the energy of $\mathrm{O}$ in an $\mathrm{O}_{2}$ gas molecule; In $\mathrm{O}_{\text {poor }}$ condition, the the $\mu$ o is given by the energy of $\mathrm{O}$ in an $\mathrm{CuO}$ lattice. 